\title{
Bounded geometry of quadrilaterals and variation of multipliers for rational maps
}

by

Kevin M. Pilgrim (Bloomington, IN)

\begin{abstract}
Let $Q$ be the unit square in the plane and $h: Q \rightarrow h(Q)$ a quasiconformal map. When $h$ is conformal off a certain self-similar set, the modulus of $h(Q)$ is bounded independent of $h$. We apply this observation to give explicit estimates for the variation of multipliers of repelling fixed points under a "spinning" quasiconformal deformation of a particular cubic polynomial.
\end{abstract}

1. Introduction. Grötzsch's length-area argument shows that if $h$ : $Q \rightarrow h(Q)$ is a side-preserving $K$-quasiconformal map between Euclidean rectangles, then

$$
\frac{\bmod (h(Q))}{\bmod (Q)} \leq \frac{\int_{Q} K_{h}(z) d A}{\int_{Q} d A}=\operatorname{average}_{Q}\left(K_{h}(z)\right),
$$

where

$$
K_{h}(z)=\frac{1+\left|\mu_{h}(z)\right|}{1-\left|\mu_{h}(z)\right|}
$$

and $\mu_{h}=h_{\bar{z}} / h_{z}$ is the complex dilatation of $h$ (see e.g. [GL, Prop. 1.4.3]). Thus, $\bmod (h(Q)) / \bmod (Q) \leq K$. The distortion of moduli may also be controlled if area $\left(\operatorname{supp}\left(\mu_{h}\right)\right) / \operatorname{area}(Q)$ is small relative to $K$. Furthermore, if $h$ is conformal off a fixed compact subset $\mathcal{M}$ of $Q$, then $\bmod (h(Q)) / \bmod (Q)$ is bounded above by a constant depending only on $\mathcal{M}$. To see this, simply compute lower bounds on the extremal lengths of horizontal and vertical path families using the Euclidean metric.

The following result, however, is somewhat surprising. It was used by Yoccoz in unpublished work proving local connectivity of the Mandelbrot set at certain parameters, and seems to have been common knowledge also to Douady, Hubbard, and McMullen at the same time. Thus, we shall refer to it as the DHMY bound.

2000 Mathematics Subject Classification: Primary 37F30; Secondary 37F10, 30F60. Research supported by Indiana University. 
Let

$$
Q=\{z \in \mathbb{C}: 0<\operatorname{Im}(z), \operatorname{Re}(z)<1\}
$$

denote the unit square in the complex plane. Subdivide $Q$ into nine congruent squares of side $1 / 3$, and denote the bottom three squares $L, M, R$, from left to right; see the upper-left image of Figure 1 (in $\S 2$ ). Let $F: \bar{L} \sqcup \bar{R} \rightarrow \bar{Q}$ be given by $F(z)=3 z$ if $z \in \bar{L}$ and $F(z)=3(z-1)+1$ if $z \in \bar{R}$. Let $M_{n}=F^{-n}(M)$ and $\mathcal{M}=\bigcup_{n} M_{n}, n \geq 0$.

Theorem 1.1 (Douady-Hubbard-McMullen-Yoccoz (DHMY) bound). Let $h: \bar{Q} \rightarrow \mathbb{C}$ be a homeomorphism onto its image which is quasiconformal on $Q$ and conformal on $Q-\mathcal{M}$. Then the modulus of the quadrilateral $h(Q)$ is bounded above and below by positive constants independent of $h$.

In $\S 2$ below we give a proof of the DHMY bound following an idea of A. Douady, and of a more general version suitable for application to dynamics. In $\S 3$ we apply this to a concrete problem concerning variation of multipliers under certain quasiconformal deformations called spins. Computer pictures aid significantly in visualizing the decomposition of the dynamical plane needed in order to apply the bound; see Figure 3.

The problem we consider is a very special case of the following general situation. For relevant definitions, see $[\mathrm{MS}]$. Let Rat ${ }_{d}$ denote the space of degree $d$ rational maps from the complex projective line $\mathbb{P}^{1}$ to itself. Let $f \in \mathrm{Rat}_{d}$ be generic and hyperbolic. Then the Teichmüller space Teich $(f)$ is isomorphic to a product $\prod_{i} \operatorname{Teich}\left(T_{i}\right)$ of classical Teichmüller spaces of punctured tori $T_{i}$ arising from attracting basins. There is a natural holomorphic map $\eta: \operatorname{Teich}(f) \rightarrow \operatorname{Rat}_{d} / \operatorname{Aut}\left(\mathbb{P}^{1}\right)$ obtained via the Measurable Riemann Mapping Theorem. The map $\eta$ is in fact the universal orbifold covering of the open subspace of Möbius conjugacy classes of maps which are quasiconformally conjugate to $f$ on all of $\mathbb{P}^{1}$. The deck group $\operatorname{Mod}(f)$ is an infinite index subgroup of the product of classical modular groups $\operatorname{Mod}\left(T_{i}\right)$ which acts on Teich $(f)$ by holomorphic isometries via precomposition of markings. By a result of Makienko ([Mak, Thm. 1]) $\operatorname{Mod}(f)$ is infinitely generated if and only if some basin attracts at least two critical points. In this case, the map $\eta$ is necessarily very wild. It is natural to ask e.g. what happens to a sequence of the form $f_{n}=\eta\left([\omega]^{n} \cdot f_{0}\right), n=0,1, \ldots$, where $[\omega] \in \prod_{i} \operatorname{Mod}\left(T_{i}\right)-\operatorname{Mod}(f)$.

Spinning. Consider the one-parameter family

$$
f_{a}(z)=\frac{1}{2} z+a z^{2}-\frac{1}{3} z^{3}, \quad a \in \mathbb{C},
$$

of normalized cubic polynomials for which the origin is an attracting fixed point of constant multiplier $1 / 2$. Here, $1 / 2$ is chosen merely for concreteness. Note that the maps $f_{ \pm a}$ are conjugate via $z \mapsto-z$. 
The basepoint $f_{0}(z)=z / 2-z^{3} / 3$ is odd, and both critical points $c_{ \pm}=$ $\pm 1 / \sqrt{2}$ lie in the immediate basin $\Omega_{0}$ of the origin. By Königs' Theorem, there is a unique holomorphic map $\phi: \Omega_{0} \rightarrow \mathbb{C}$ such that $\phi \circ f(z)=\phi(z) / 2$ and normalized so that $\phi^{\prime}(0)=1$. Let $T$ be the torus $(\mathbb{C}-\{0\}) /\langle w \mapsto w / 2\rangle$, let $p: \mathbb{C}-\{0\} \rightarrow T$ denote the projection, and put $S=T-(p \circ \phi)\left(c_{ \pm}\right)$. Then $S$ is a twice-punctured torus. Let $A^{ \pm}$be the image of the domain $\{w \in \mathbb{C}-\{0\}: \pm \operatorname{Arg}(w) \in(\pi / 4,3 \pi / 4)\}$ under the projection $p$. Then $A^{ \pm}$ is a pair of disjoint, symmetric, open annuli compactly contained in $S$.

Let $A$ be the annulus $[0,2 \pi] \times[0,1]$ where the first coordinate is read modulo $2 \pi$, equipped with the counterclockwise orientation inherited from $\mathbb{R}^{2}$. The standard affine positive/negative Dehn twist is given by $(\theta, t) \mapsto(\theta \pm$ $2 \pi t, t)$. Thus, the image of a curve of the form $\left\{\theta_{0}\right\} \times[0,1]$ under a positive/negative Dehn twist bends right/left as the curve is traversed, independent of orientation of the curve.

Let $\omega: S \rightarrow S$ be the homeomorphism which is the identity off $A^{ \pm}$, and is the standard affine positive/negative Dehn twist on $A^{ \pm}$. The homeomorphism $\omega$ is called a spin. See [PT], [GK], [Cui] for spinning in the context of rational maps. We consider the sequence $\eta\left([\omega]^{n} . f_{0}\right)$. Recalling that the action is by precomposition of markings, note that the image of the basepoint $S$ under the action of $[\omega]^{n}$ is represented by $\left(\omega^{-n}: S \rightarrow S\right)$ where $\omega^{-1}$ is now a negative Dehn twist on $A^{+}$and a positive Dehn twist on $A^{-}$. Let $\nu_{n}$ denote the complex dilatation of $\omega_{n}=\omega^{\circ-n}$ on $S$, and let $\mu_{n}$ be the Beltrami differential on $\mathbb{C}$ given by $(p \circ \phi)^{*}\left(\nu_{n}\right)$ on $\Omega_{0}$ and zero elsewhere.

By the Measurable Riemann Mapping Theorem, there is a unique quasiconformal map $H_{n}: \mathbb{C} \rightarrow \mathbb{C}$ whose complex dilatation is $\mu_{n}$ a.e., normalized by $H_{n}(0)=0$ and $H(z)=z+O(1)$ near infinity. Since the maps $\omega_{n}$ extend to maps of $T, H_{n} \circ f_{0} \circ H_{n}^{-1}=f_{a_{n}}$ for some $a_{n} \in \mathbb{C}$. Since $\mu_{n}(\bar{z})=\overline{\mu_{n}(z)}$, we have $a_{n} \in \mathbb{R}$, and in fact $a_{n}>0$. For each $n$, the map $f_{a_{n}}$ has a pair $v_{n}^{+}, v_{n}^{-}$ of repelling fixed points in the upper and lower half-planes, respectively. Let $\lambda_{n}^{ \pm}=f_{a_{n}}^{\prime}\left(v_{n}^{ \pm}\right)$. When $n=0, \lambda_{0}^{ \pm}=2$. There are two unique components $\widetilde{A}^{ \pm}$ of $(p \circ \phi)^{-1}\left(A^{ \pm}\right)$which join the origin to $v_{0}^{ \pm}$.

Theorem 1.2. As $n \rightarrow \infty$,

$$
\lambda_{n}^{ \pm}=\exp \left(-2 \pi i \frac{\tau_{0}}{ \pm n \tau_{0}+1}\right)+O\left(\frac{1}{n^{2}}\right)=1 \mp 2 \pi i \frac{1}{n}+O\left(\frac{1}{n^{2}}\right)
$$

where $\tau_{0}=(i \ln 2) / 2 \pi$. More precisely, for sufficiently large $n$, the indices $\iota_{n}^{ \pm}=1 /\left(1-\lambda_{n}^{ \pm}\right)$satisfy

$$
\iota_{n}^{ \pm}= \pm \frac{n}{2 \pi i}+\left(\frac{1}{2}-\frac{1}{\ln 2}\right)+O(1)
$$

where the real part of the constant is strictly less than $1 / \ln 2$. 
An easy application of the Holomorphic Fixed Point Formula ([Mil, Thm. 12.4]) and a bit of algebra yields

Corollary 1.1. As $n \rightarrow \infty, f_{a_{n}} \rightarrow f_{a}$, where $a=\sqrt{6} / 3$. More precisely,

- $a_{n}=\frac{\sqrt{6}}{3}-\frac{\pi \sqrt{6}}{3} \frac{1}{n^{2}}+O\left(\frac{1}{n^{3}}\right)$,

- the repelling fixed points $v_{n}^{ \pm}$collide to a common parabolic fixed point of $f_{a}$ of multiplier 1 .

In particular, $f_{n} \not f f$ horocyclically in the sense of McMullen [McM], since $\tau_{0} /\left( \pm n \tau_{0}+1\right) \rightarrow 0$ along a horocycle in the upper half-plane model of the hyperbolic plane. A non-quantitative version of this corollary was obtained in much greater generality in [PT], using indirect methods.

REMARKS. Versions of the DHMY bound appear in several other places. In [Haï, Prop. 3.6] it is stated more or less as above; the proof given there is very different. There, the DHMY bound is used to prove uniform moduli of continuity of quasiconformal conjugacies under "pinching" deformations. Spinning, however, produces parabolic implosions and equicontinuity fails. G. Z. Cui [Cui], J. Kahn [K], and J. Rivera-Letelier [R] have more general rigidity statements. Cui's proof uses some novel estimates for conformal distortion, while the latter two rely on certain Sobolev estimates.

Acknowledgements. I thank A. Douady for the idea of the proof of Theorem 1.1, L. Keen for useful conversations, and Tan L. for a critical reading of a first draft and communicating A. Douady's proof. I also thank P. Haïssinsky and M. Shishikura for pointing out errors in earlier versions.

2. Proof of DHMY bound. We prove the DHMY bound first, followed by a quasiconformally distorted version suitable for our application.

Let $\Gamma_{\text {hor/ver }}$ denote the families of rectifiable paths joining respectively the vertical and horizontal sides of $Q$. It is enough to establish lower bounds on the extremal lengths $\Lambda\left(h\left(\Gamma_{\text {hor } / v e r}\right)\right)$ of the horizontal and vertical path families $h\left(\Gamma_{\text {hor } / \text { ver }}\right)$ for $h(Q)$, since these quantities are reciprocals of one another. Let $U_{0}=Q \cap\{\operatorname{Im}(z)>1 / 3\}$, let $U_{n}=F^{-n}\left(U_{0}\right), n=1,2, \ldots$, and let $\mathcal{U}=\operatorname{int}\left(\overline{\bigcup_{n \geq 0} U_{n}}\right)$. Since $h$ is conformal on $\mathcal{U}, \Lambda\left(h\left(\Gamma_{\text {hor } / \text { ver }}\right)\right)$ is bounded below by

$$
\sup _{\varrho} \frac{\left(\inf _{\gamma \in \Gamma_{\text {hor } / \text { ver }}} \int_{\gamma}|d z|\right)^{2}}{\int_{Q} \varrho^{2}|d z|^{2}},
$$

where $\varrho \geq 0$ is square-integrable and supported on $\mathcal{U}$. Letting $\varrho \equiv 1$ on $\mathcal{U}$ shows that $\Lambda\left(h\left(\Gamma_{\text {ver }}\right)\right) \geq 2 / 3$. 

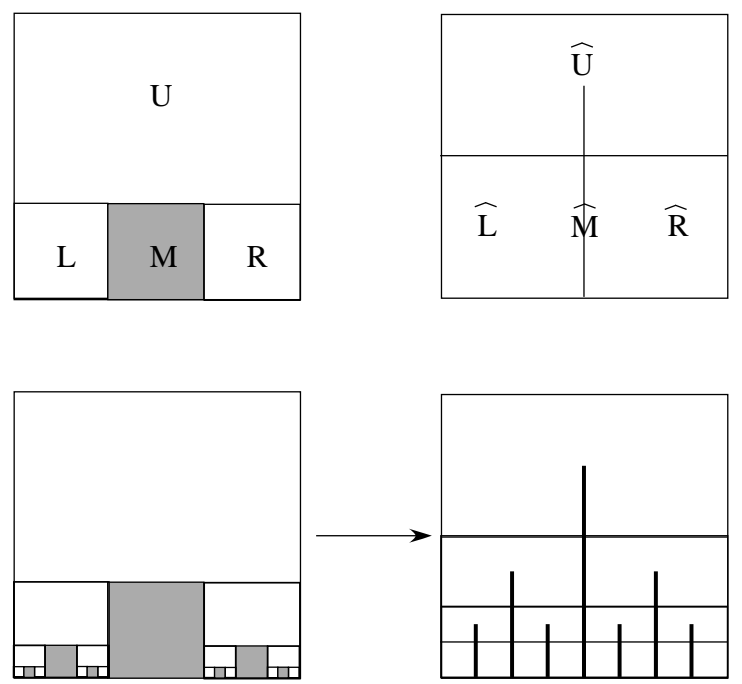

Fig. 1. $\mathcal{U}$ is the complement of the gray region at lower left. $\widehat{\mathcal{U}}$ is the complement of the vertical segments at the lower right. The map $\psi$ sends the unit square continuously to itself so that $\psi(\mathcal{U})=\widehat{\mathcal{U}}$ and $\left.\psi\right|_{\mathcal{U}}$ is $K$-qc.

To find a lower bound for $\Lambda\left(h\left(\Gamma_{\text {hor }}\right)\right)$, we use an idea of A. Douady; see Figure 1. By postcomposing $h$ with a Riemann map to a rectangle followed by a conformal affine map, we may assume $h$ is a side-preserving homeomorphism from $\bar{Q}$ to the rectangle $[0, l] \times[0,1]$, so that $\Lambda\left(h\left(\Gamma_{\text {hor }}\right)\right)=l=$ area $(h(Q))$. To bound the area from below, we claim there exists a continuous map $\psi: \bar{Q} \rightarrow \bar{Q}$ such that $\psi$ collapses each component of $M_{n}$ to a vertical segment of the form $\left\{i / 2^{-(n+1)}\right\} \times\left[0, \frac{3}{4} \cdot 2^{-n}\right]$ and which is $K$-quasiconformal on the complement of $\mathcal{U}$ of the closure of $\mathcal{M}$; see Figure 1. Assuming this claim for the moment, we reason as follows. Let $\widehat{\mathcal{U}}=\psi(\mathcal{U})$.

Let $H=\left.h \circ \psi^{-1}\right|_{\hat{\mathcal{U}}}$. Then $H$ is $K$-quasiconformal on $\widehat{U}$. Thus

$$
\begin{aligned}
\operatorname{area}(h(Q)) & \geq \operatorname{area}(h(\mathcal{U}))=\operatorname{area}(H(\widehat{\mathcal{U}}))=\int_{\widehat{\mathcal{U}}} \operatorname{Jac}(H) d A \\
& \geq \frac{1}{K} \iint_{\widehat{\mathcal{U}}}\left|\frac{\partial H}{\partial y}\right|^{2} d y d x \quad \text { since } H \text { is } K \text {-qc } \\
& \geq \frac{1}{K}\left(\iint_{\widehat{\mathcal{U}}}\left|\frac{\partial H}{\partial y}\right| d y d x\right)^{2} / \iint_{\widehat{\mathcal{U}}} 1 d y d x \quad \text { by Schwarz' } \leq .
\end{aligned}
$$

Each vertical segment of the form $\{x\} \times[0,1]$, where $x \neq i / 2^{k}, i, k \in \mathbb{N}$, is mapped by $H$ to a continuous path joining the top and bottom edges of the rectangle $h(\bar{Q})=[0, l] \times[0,1]$ and thus has length $\geq 1$. By Fubini's Theorem the integral in the numerator is therefore at least one. The domain $\widehat{\mathcal{U}}$ has area 1 , so area $(h(Q)) \geq 1 / K$. 
To find $\psi$ is straightforward but tedious; we merely sketch the idea. Let $\widehat{L}, \widehat{R}$ be as shown in Figure 1. Combinatorially, the map $\widehat{F}: \overline{\widehat{L}} \sqcup \overline{\widehat{R}} \rightarrow \bar{Q}$ given by $\widehat{F}(z)=2 z$ on $\widehat{\widehat{L}}$ and by $2(z-1)+1$ on $\widehat{\widehat{R}}$ is the same as $F$.

Let $\widehat{U}$ be the slit rectangle at the top of Figure 1. Let $\psi$ send the top, left, and right sides of $\partial U$ to the corresponding sides of $\partial \widehat{U}$ via affine maps. Fold the middle third of the bottom to the slit, and send the bottom left third and right third via pulling back so that $\psi$ commutes with the dynamics there. Extend $\psi$ to a continuous map $\psi: \bar{U} \rightarrow \overline{\widehat{U}}$ via piecewise-linear maps on $U$. The maps on the sides of $M$ will be determined by the dynamics; extend to the interior of $M$ by a continuous collapsing map. By insisting that $\psi$ be continuous and conjugate $F$ to $\widehat{F}, \psi$ is determined on all of $\bar{Q}$. Since both $F$ and $\widehat{F}$ are conformal, $\psi$ will be $K$-qc on $\mathcal{U}$.

In practice, the setup in Theorem 1.1 must be applied in a slightly distorted context.

Assumptions.

(1) $Q^{\prime}$ is a quadrilateral bounded by a quasicircle. Suppose $Q^{\prime}$ is subdivided into nine quadrilaterals, each bounded by quasicircles, in a manner combinatorially the same as that described in $\S 1$. Let $L^{\prime}, M^{\prime}, R^{\prime}, U^{\prime}$ be defined similarly.

(2) Suppose $U_{L}^{\prime}, U_{R}^{\prime}, V^{\prime}$ are open Jordan domains such that $\overline{L^{\prime}} \subset U_{L}^{\prime}$, $\overline{R^{\prime}} \subset U_{R}^{\prime}, \overline{Q^{\prime}} \subset V^{\prime}$, and that $U_{L}^{\prime}$ and $U_{R}^{\prime}$ are compactly contained in $V^{\prime}$. Assume we are given a proper holomorphic map $F^{\prime}: U_{L}^{\prime} \sqcup$ $U_{R}^{\prime} \rightarrow V^{\prime}$, univalent on each component, such that $F\left(\overline{L^{\prime}}\right)=\overline{Q^{\prime}}=$ $F\left(\overline{R^{\prime}}\right)$. It follows that the bottom corners of $Q^{\prime}$ are repelling fixed points of $F^{\prime}$. Let $\mathcal{M}^{\prime}=\bigcup_{n \geq 0}\left(F^{\prime}\right)^{-n}\left(M^{\prime}\right)$. We also assume that there exists an anticonformal involution of $V^{\prime}$ sending $Q^{\prime}$ to itself which interchanges $L^{\prime}$ and $R^{\prime}$, sends $U^{\prime}, M^{\prime}$ to themselves, and commutes with $F^{\prime}$ (this is the analog of reflection in the line $x=1 / 2$ for $Q$ ).

(3) The four arcs comprising $\partial Q$ are analytic.

TheOREM 2.1. Under assumptions (1)-(3) above, let $h^{\prime}: \overline{Q^{\prime}} \rightarrow \mathbb{C}$ be a homeomorphism onto its image which is quasiconformal on $Q^{\prime}$ and conformal on $Q^{\prime}-\mathcal{M}^{\prime}$. Then the modulus of $h^{\prime}\left(Q^{\prime}\right)$ is bounded above and below by positive constants independent of $h^{\prime}$.

Proof. It suffices to show the existence of a side-preserving quasiconformal map $\phi: Q \rightarrow Q^{\prime}$ sending $\mathcal{M}$ to $\mathcal{M}^{\prime}$. The only difficult point is that we may no longer use a piecewise-affine extension to the interior to get a qc map - we must be careful, since the gluing of two quasisymmetric maps at a point need not be quasisymmetric. 
Assume first that there exists a side-preserving $K$-quasiconformal map $\phi_{0}: Q \rightarrow Q^{\prime}$ with the following properties:

- $\phi_{0}$ sends $U, L, M, R$ to $U^{\prime}, L^{\prime}, M^{\prime}, R^{\prime}$,

- $\phi_{0} \circ F=F^{\prime} \circ \phi_{0}$ on the left, right, and top sides of $L$ and $R$.

For $n \geq 0$, let $U_{n}=F^{-n}(U), M_{n}=F^{-n}(M)$, and define $U_{n}^{\prime}$, etc. similarly. Inductively define $\phi_{n+1}: Q \rightarrow Q^{\prime}$ by setting $\phi_{n+1}=\phi_{n}$ on $\overline{U_{n} \cup M_{n}}$ and by $\left(F^{\prime}\right)^{-1} \circ \phi_{n} \circ F$ on $\overline{Q^{\prime}-U_{n} \cup M_{n}}$. For each $n, \phi_{n}$ is a side-preserving $K$-quasiconformal map from $Q$ to $Q^{\prime}$. By compactness properties of $K$ quasiconformal mappings, after passing to a subsequence, there is a limit $\phi: Q \rightarrow Q^{\prime}$ which conjugates $F$ to $F^{\prime}$, hence sends $\mathcal{M}$ to $\mathcal{M}^{\prime}$.

We now show the existence of $\phi_{0}$. We shall use repeatedly the fact that quasisymmetric maps between quasicircles extend over the complementary components to quasiconformal maps.

Consider the quotient tori $T_{L}, T_{R}, T_{L}^{\prime}, T_{R}^{\prime}$ for the repelling fixed points of $F$ and $F^{\prime}$; see Figure 2.

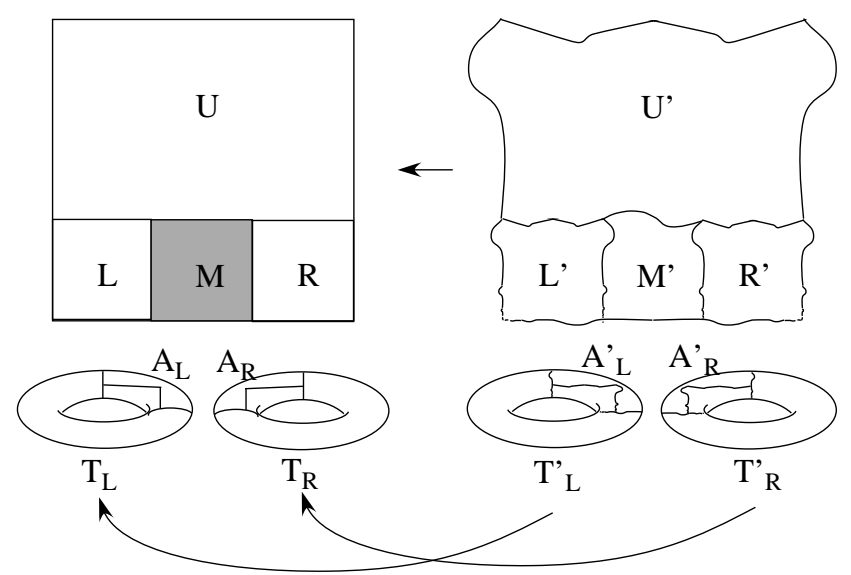

Fig. 2. Finding $\phi_{0}$. Send $A_{L / R}^{\prime}$ to $A_{L / R}$ via a qc map which sends the crosscut curves to curves homotopic to crosscut curves.

The anticonformal involutions of $Q, Q^{\prime}$ descend to anticonformal isomorphisms $T_{L} \leftrightarrow T_{R}$ and $T_{L}^{\prime} \leftrightarrow T_{R}^{\prime}$. The domains $L, R, L^{\prime}, R^{\prime}$ project to annuli $A_{L}, A_{R}$, etc. bounded by real analytic closed curves. Each such annulus contains a distinguished piecewise-analytic crosscut curve (the projection of the union of the top and side of $L, R$ ) which joins the two boundary components. Moreover, the projection of the bottom of $M^{\prime}$ (and of $M$ ) project to distinguished segments on the boundary components of $A_{L}, A_{R}$, etc.

Condition (3) implies that there exists a $C^{1}$, hence qc map $T_{L}^{\prime} \rightarrow T_{L}$ sending $A_{L}^{\prime} \rightarrow A_{L}$, mapping the distinguished crosscut curves to curves homotopic to the corresponding crosscut curves, and sending the distinguished 
segments to the corresponding segments. By conjugating with the anticonformal involution, we get a similar map $T_{R}^{\prime} \rightarrow T_{R}$.

Lifting to the dynamical plane, we obtain a continuous map defined on the bottom, left, and right sides of $Q^{\prime}$ to the corresponding sides of $Q$, which commutes with the dynamics on the left and bottom (respectively, right and bottom) of $L^{\prime}$ and $R^{\prime}$, and which sends the corners of $L^{\prime}, R^{\prime}$ lying on $\partial Q^{\prime}$ to the corresponding corners in $\partial Q$. The resulting map is quasisymmetric since it arises as the boundary values of a locally defined qc map. This map extends to a quasisymmetric map $\partial Q^{\prime} \rightarrow \partial Q$, which in turn extends to a quasiconformal map $Q^{\prime} \rightarrow Q^{\prime}$ which we denote by $\phi_{-1}$.

Set $\left.\phi_{0}\right|_{L^{\prime} / R^{\prime}}=F_{L / R}^{-1} \circ \phi_{-1} \circ F_{L^{\prime} / R^{\prime}}^{\prime}$. The resulting map extends, using the boundary values of $\phi_{-1}$, continuously to $L^{\prime} \sqcup R^{\prime} \bigcup \partial Q^{\prime}$ such that the restriction to $\partial Q^{\prime}$ is quasisymmetric. Extend $\phi_{0}$ over the complement of $\overline{Q^{\prime}}$ in the Riemann sphere to a quasiconformal map of the quasidisk $\left(\widehat{\mathbb{C}}-\overline{Q^{\prime}}\right) \cup$ $\overline{L^{\prime}} \cup \overline{R^{\prime}}$ onto $(\widehat{\mathbb{C}}-\bar{Q}) \cup \bar{L} \cup \bar{R}$. It remains only to extend $\phi_{0}$ over $U^{\prime} \cup M^{\prime}$ so that $\phi_{0}\left(U^{\prime}\right)=U$ and $\phi_{0}\left(M^{\prime}\right)=M$. The Corollary to Lemma 2.1 below says precisely that this is possible, and the proof is complete.

Lemma 2.1. Suppose two Jordan domains $V_{ \pm} \subset \mathbb{C}$ share a common boundary arc, and that each component of $\widehat{\mathbb{C}}-\left(\partial V_{+} \cup \partial V_{-}\right)$is a quasicircle. Let $f_{+}: \widehat{\mathbb{C}} \rightarrow \widehat{\mathbb{C}}$ be a quasiconformal homeomorphism for which $f\left(\Delta_{+}\right)=V_{+}$. Then there is a quasiconformal homeomorphism $f: \widehat{\mathbb{C}} \rightarrow \widehat{\mathbb{C}}$ such that $f\left(\Delta_{ \pm}\right)=V_{ \pm}$and $f=f_{+}$on $\Delta_{+}$.

Proof. Recall that $\Delta$ denotes the open unit disk in $\mathbb{C}$, and $\Delta_{ \pm}$the upper and lower halves. Then $\Delta_{ \pm}$are both quasidisks, i.e. each is the image of $\Delta$ under a quasiconformal homeomorphism $g_{ \pm}$of all of $\mathbb{C}$, and without loss of generality we may assume that $g_{+}$and $g_{-}$send the lower and upper semicircles, respectively, onto the interval $[-1,1]$.

Let $f_{+}$be a quasiconformal map of the plane sending $\Delta_{+}$to $V_{+}$. The composition $\left.f_{-}^{-1} \circ f_{+}\right|_{[-1,1]}$ is a homeomorphism of the interval $[-1,1]$ to itself. The composition $g_{-}^{-1} \circ\left(\left.f_{-}^{-1} \circ f_{+}\right|_{[-1,1]}\right) \circ g_{-}$is a quasisymmetric map of the upper semicircle to itself. Extend this map over the lower semicircle by complex conjugation. Lemma 5.1 of [Leh] implies that the resulting map $h$ : $\partial \Delta \rightarrow \partial \Delta$ is quasisymmetric. Applying e.g. the Ahlfors-Beurling extension, we obtain a quasiconformal extension $H: \Delta \rightarrow \Delta$.

Let $f: \Delta \rightarrow \mathbb{C}$ be given by $f_{+}$on $\Delta_{+} \cup(-1,1)$ and by $f_{-} \circ g_{-} \circ H \circ g_{-}^{-1}$ on $\Delta_{+} \cup(-1,1)$. The map $f$ is a homeomorphism on $\Delta$, and quasiconformal on $\Delta_{+}$and $\Delta_{-}$, hence is quasiconformal on $\Delta$ by the removability properties of analytic arcs for qc maps. The map $f$ extends to a homeomorphism of $\bar{\Delta}$ sending the quasicircle $\partial \Delta$ to a quasicircle, by assumption. It follows from [LV, Thm. II.8.2] that $f$ extends to a quasiconformal map on a neighborhood 
of $\bar{\Delta}$, which then by [LV, Thm. II.8.1] extends to a quasiconformal map on the entire plane.

Corollary 2.1. Suppose two Jordan domains $V_{ \pm} \subset \mathbb{C}$ share a common boundary arc, and that each component of $\widehat{\mathbb{C}}-\left(\partial V_{+} \cup \partial V_{-}\right)$is a quasicircle. Let $W=\widehat{\mathbb{C}}-\left(\bar{V}_{+} \cup \bar{V}_{-}\right)$, and suppose $f: \widehat{\mathbb{C}}-\bar{\Delta} \rightarrow W$ is a quasiconformal map. Then there exists a quasiconformal extension of $f$ to the whole sphere such that $f\left(\Delta_{ \pm}\right)=V_{ \pm}$.

The proof follows by letting $W$ correspond to $V_{+}$in Lemma 2.1.

3. Proof of Theorem 1.2. We prove the bound for $\lambda_{n}^{+}$; the other is similar. Let $\phi^{+}:(\mathbb{C}, 0) \rightarrow\left(\mathbb{P}^{1}, v_{0}^{+}\right)$denote the Königs linearizing map for the repelling fixed point $v_{0}^{+}$, and let $p^{+}: \mathbb{C}-\{0\} \rightarrow T_{0}^{+}$be projection to the quotient torus $T_{0}^{+}$. Identify $T_{0}^{+}$with $\mathbb{C} /\left\langle z \mapsto z+1, z \mapsto z+\tau_{0}\right\rangle$ where $\tau_{0}=$ $(\ln 2) i / 2 \pi$. Let $\alpha, \beta$ be generators of $\pi_{1}\left(T_{0}^{+}, 0\right)$ corresponding to translation by 1 and translation by $\tau_{0}$, respectively. Thus, a lift of $\alpha$ under $p^{+} \circ \phi^{+}$goes once around $v_{0}^{+}$counterclockwise, while a lift of $\beta$ points in toward $v_{0}^{+}$.

Let $\mathbb{H}$ denote the upper half-plane and $\mathcal{T}$ the Teichmüller space of complex tori modeled on $T_{0}^{+}$. A point of $\mathcal{T}$ is represented by a complex torus $T^{+}=\mathbb{C} /\langle 1, \tau\rangle, \tau \in \mathbb{H}$, equipped with a quasiconformal homeomorphism $h: T_{0}^{+} \rightarrow T^{+}$such that a lift of $h$ to $\mathbb{C}$ conjugates translation by 1 and by $\tau_{0}$ to translation by 1 and by $\tau$, respectively. The map sending a marked torus $\left(h: T_{0}^{+} \rightarrow T^{+}\right)$to the point $\tau$ is an isomorphism between $\mathcal{T}$ and the upper half-plane $\mathbb{H}$ and is an isometry with respect to the Teichmüller and hyperbolic metrics.

The mapping class group acts on $\mathcal{T}$ by precomposition of markings. Suppose $\omega: T_{0}^{+} \rightarrow T_{0}^{+}$is an arbitrary quasiconformal map. Let $[\omega]$ denote the corresponding mapping class element and $\omega_{*}$ the induced linear map on $H_{1}\left(T_{0}^{+}, \mathbb{Z}\right)$, equipped with the ordered basis $[\alpha, \beta]$. Then

$$
[\omega] \cdot \tau=\frac{a \tau+b}{c \tau+d}
$$

where $\left(\begin{array}{ll}a & b \\ c & d\end{array}\right)$ is the matrix for $\omega_{*}^{-1}$. If $\omega$ is a positive Dehn twist in an annulus with core curve $\beta$, then $\omega_{*}^{-1}$ sends $\beta \mapsto \beta$ and $\alpha \mapsto \alpha+\beta$ on homology and so $[\omega] . \tau=\tau /(\tau+1)$.

Now let $\omega: S \rightarrow S$ be a spin on the quotient twice-punctured torus $S$ corresponding to the attractor at the origin, $\omega_{n}=\omega^{-n}$, and let $\mu_{n}$ be the Beltrami differential of $\omega_{n}$ lifted to the sphere (recall the discussion in $\S 1$ ). The Beltrami differentials $\mu_{n}$ are $f_{0}$-invariant, hence descend to Beltrami differentials on $T_{0}^{+}$which we denote by $\mu_{n}^{+}$. Then the $\mu_{n}^{+}$determine tori

$$
T_{n}^{+}=\mathbb{C} /\left\langle z \mapsto z+1, z \mapsto z+\tau_{n}^{+}\right\rangle, \quad \tau_{n}^{+} \in \mathbb{H},
$$


and quasiconformal maps $h_{n}^{+}: T_{0}^{+} \rightarrow T_{n}^{+}$, i.e. points $\tau_{n}^{+}$in Teichmüller space. The multipliers satisfy $\lambda_{n}^{+}=\exp \left(-2 \pi i \tau_{n}^{+}\right)$.

The map $\left(p^{+} \circ \phi^{+}\right) \circ\left(\left.p \circ \phi\right|_{A^{+}}\right)^{-1}: A^{+} \rightarrow A$ is a conformal isomorphism from the annulus $A^{+}$in the quotient torus $S$ for the attractor at the origin to an annulus $A$ in the quotient torus $T_{0}^{+}$for the repellor at $v_{0}^{+}$. Write $\mu_{n}^{+}=\mu_{n}^{A}+\kappa_{n}$, where $\kappa_{n} \equiv 0$ on $A$. The key observation is that the Beltrami differential $\mu_{n}^{A}$ agrees with that of $\omega_{n}^{+}=\omega^{\circ-n}: T_{0}^{+} \rightarrow T_{0}^{+}$, where $\omega$ is a positive Dehn twist on $A$. Let $t_{n}^{+}=[\omega]^{-n} \cdot \tau_{0}=\tau_{0} /\left(n \tau_{0}+1\right) \in \mathbb{H}$.

Lemma 3.1. There is a constant $C_{0}$ such that for all $n \in \mathbb{N}$,

$$
d\left(t_{n}^{+}, \tau_{n}^{+}\right) \leq C_{0},
$$

where $d$ is the hyperbolic (Teichmüller) distance.

Given the lemma, let us now prove the theorem. The composition

$$
\mathbb{H} \stackrel{\lambda=\exp (-2 \pi i \tau)}{\longrightarrow}\{\lambda:|\lambda|>1\} \stackrel{\iota=1 /(1-\lambda)}{\longrightarrow}\{\iota: \operatorname{Re}(\iota)<1 / 2\}-\{0\}
$$

is an isometry with respect to the hyperbolic metrics. On the last domain, for large imaginary values, the hyperbolic metric is comparable to the metric $d x /(x-1 / 2)$. This observation and a routine power series computation yields the second conclusion.

To prove the first estimate, note that the point on a hyperbolic circle in $\mathbb{H}$ which is Euclidean furthest away from the center lies directly above the center. Thus

$$
d(z, w) \leq C \Rightarrow|z-w| \leq\left(e^{C}-1\right) \operatorname{Im}(z)
$$

and so

$$
\left|t_{n}^{+}-\tau_{n}^{+}\right| \leq\left(e^{C_{0}}-1\right) \operatorname{Im}\left(t_{n}^{+}\right) .
$$

Putting $C_{1}=e^{C_{0}}-1$ and substituting $t_{n}^{+}=\tau_{0} /\left(n \tau_{0}+1\right)$ yields

$$
\left|t_{n}^{+}-\tau_{n}^{+}\right| \leq C_{1} \frac{\frac{\ln 2}{2 \pi}}{n^{2}\left(\frac{\ln 2}{2 \pi}\right)^{2}+1} .
$$

As $n \rightarrow \infty$, both $t_{n}^{+}$and $\tau_{n}^{+}$tend to zero, where the map $\tau \mapsto \exp (-2 \pi i \tau)$ is nearly a Euclidean affine map with derivative $-2 \pi i$. Since $\lambda_{n}^{+}=$ $\exp \left(-2 \pi i \tau_{n}\right)$, this yields after cancelling $2 \pi$ 's

$$
\left|\exp \left(-2 \pi i t_{n}^{+}\right)-\lambda_{n}^{+}\right|<C_{2} \frac{\ln 2}{n^{2}\left(\frac{\ln 2}{2 \pi}\right)^{2}+1}=O\left(\frac{1}{n^{2}}\right) .
$$

Proof of Lemma 3.1. In $\mathcal{T}$, the point $\tau_{n}^{+}$is represented by $h_{n}^{+}: T_{0}^{+} \rightarrow T_{n}^{+}$, while $t_{n}^{+}$is represented by $\omega_{n}^{+}: T_{0}^{+} \rightarrow T_{0}^{+}$. By construction, $h_{n}^{+} \circ \omega_{n}^{-1}: T_{0}^{+} \rightarrow$ $T_{n}^{+}$is a quasiconformal map which is holomorphic off a subset $\mathcal{K}$ which is independent of $n$. 
Let $h: T_{0}^{+} \rightarrow T^{+}$be an arbitary quasiconformal mapping whose dilatation $\mu$ is supported on $\mathcal{K}$. By the definition of Teichmüller distance, it suffices to show that there are lower bounds, independent of $h$, on the moduli of annuli in $h\left(T_{0}^{+}\right)$whose core curves are homotopic to $h(\alpha)$ and to $h(\beta)$. The lower bound for $h(\beta)$ is immediate from the Grötzsch inequality, since $\mu=0$ on $A$ by hypothesis.

Let $\widetilde{\mu}$ be the Beltrami differential given by $\left(p^{+} \circ \phi^{+}\right)^{*}(\mu)$ on $\Omega_{0}$ and zero elsewhere. Then $\widetilde{\mu}$ is supported on $\widetilde{\mathcal{K}}=\left(p^{+} \circ \phi^{+}\right)^{-1}(\mathcal{K})$, which is the grand orbit of $\widetilde{A}^{-1}$. Let $H_{\widetilde{\mu}}: \mathbb{P}^{1} \rightarrow \mathbb{P}^{1}$ be a straightening map for $\widetilde{\mu}$.

Let $\mathcal{D}$ be an annulus in the dynamical plane which is a fundamental domain for the action of $f_{0}$ near $v_{0}^{+}$. Finding a lower bound on the modulus of an annulus in $h\left(T_{0}^{+}\right)$about $h(\alpha)$ is equivalent to finding a lower bound on the extremal length of the path family joining the boundary components of $H_{\widetilde{\mu}}(\mathcal{D})$.

It will be convenient to deal with a more symmetric object. Let $s(z)=$ $1 / \bar{z}$ denote inversion in the unit circle. Let $\Phi:(\Delta, 0) \rightarrow\left(\Omega_{0}, 0\right)$ be the unique Riemann map for which $\Phi^{\prime}(0)>0$. Then $\Phi$ conjugates $f_{0}$ on $\Omega_{0}$ to the Blaschke product

$$
B_{0}(z)=-z \frac{z^{2}-a^{2}}{1-a^{2} z^{2}}, \quad a=\frac{1}{\sqrt{2}} .
$$

By reflection, $\Phi$ extends to a quasiconformal conjugacy in a neighborhood of the Julia set. It suffices to prove the analogous result for $B_{0}$, where now the Beltrami differential $\mu$ is supported on the $s$-symmetric set $\widetilde{\mathcal{K}} \cup s(\widetilde{\mathcal{K}})$, but where we do not assume that $\widetilde{\mu}=s^{*} \widetilde{\mu}$.

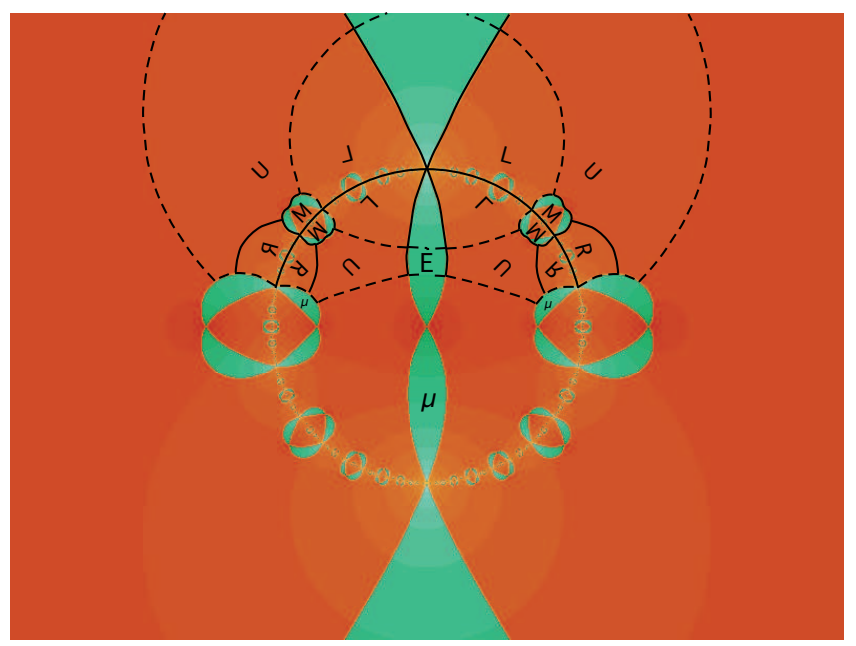

Fig. 3. Four copies of $Q$ near the repelling fixed point $v_{0}^{+}$. The Beltrami differential $\widetilde{\mu}$ is supported on the grand orbit of $\widetilde{A}^{-}$, indicated by the letter $\mu$. 
Consider the dynamical plane for $B_{0}$; see Figure 3 . It is easy to prove the existence of a quadrilateral $Q^{\prime}$ and subdomains $U^{\prime}, L^{\prime}, M^{\prime}, R^{\prime}$ as in Theorem 2.1 in e.g. the upper right-hand corner of the figure. (The anticonformal involution comes from the fact that $B_{0} \mid Q^{\prime}$ is univalent, and the image $B_{0}\left(Q^{\prime}\right)$ is symmetric with respect to the real axis.) The domains will be quasicircles: since the annuli $A^{ \pm}$can be fattened slightly, arcs which meet at the Julia set or at a point in the grand orbit of the origin do so in definite angles, while the remaining corners are intersections of analytic arcs meeting transversely.

Let

$$
\mathcal{R}=\operatorname{int}\left(\operatorname{closure}\left(Q^{\prime} \cup s\left(Q^{\prime}\right) \cup r\left(Q^{\prime}\right) \cup r s\left(Q^{\prime}\right) \cup E \cup s(E)\right)\right)
$$

where $E$ is the fundamental domain for the restriction of $B_{0}$ to the lift $\widetilde{A}^{+}$ indicated in the figure. Then $\mathcal{R}$ is an annulus surrounding $v_{0}^{+}$. A fundamental domain $\mathcal{D}$ for the action of $B_{0}$ near $v_{0}^{+}$is the region between the dashed curves in Figure 3. Combinatorially, the situation is as depicted in Figure 4.

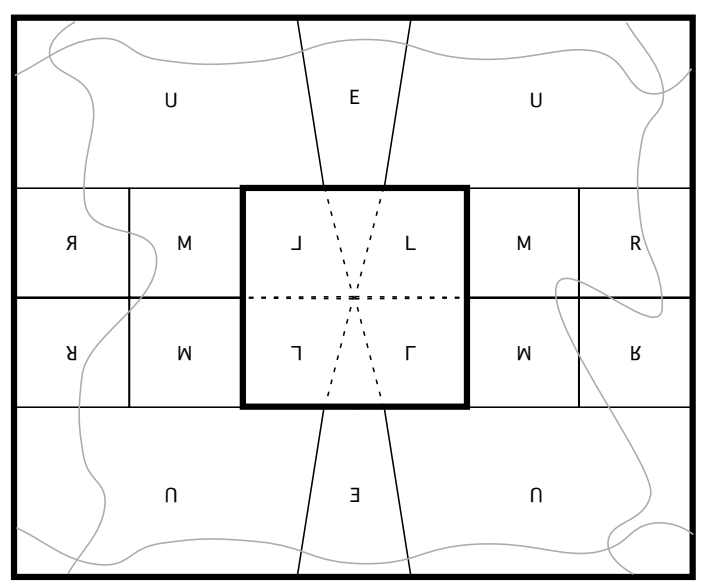

Fig. 4. An annulus surrounding the repelling fixed point is decomposed combinatorially as shown above. The grey curves are typical elements of the path families $\Gamma_{h / v}^{ \pm}$.

To complete the argument, we use an observation of G. Cui. As suggested in Figure 4, let $\Gamma_{\mathrm{h}}^{+}$consist of those paths lying in the rectangle which is the union of the top two copies of $U$ and the top copy of $E$, and which join the horizontal sides of this rectangle. Let $\Gamma_{\mathrm{h}}^{-}$be the corresponding family in the bottom half. Let $\Gamma_{\mathrm{v}}^{+}$consist of those paths lying in the rectangle which is the union of the right copies of $U, M, R$ and which join the extreme top and bottom sides of this rectangle. Let $\Gamma_{\mathrm{v}}^{-}$be the corresponding family in the left half.

Since $\widetilde{\mu}=0$ on $U \cup E$, the extremal lengths of $h\left(\Gamma_{\mathrm{h}}^{ \pm}\right)$in the annulus $h(\mathcal{D})$ are independent of $\mu$. By the DHMY bound and the subadditivity properties 
of extremal length, the extremal lengths of $h\left(\Gamma_{\mathrm{v}}^{ \pm}\right)$are bounded from above independent of $\mu$. Thus

$$
\max \left\{\Lambda\left(h\left(\Gamma_{\mathrm{h}}^{ \pm}\right)\right), \Lambda\left(h\left(\Gamma_{\mathrm{v}}^{ \pm}\right)\right)\right\} \leq k
$$

for some constant $k$.

Let $\Gamma$ now be the family of paths $\gamma$ in $\mathcal{D}$ joining the inner and outer boundary components of $\mathcal{D}$. Given any $\gamma$, among the four families $\Gamma_{\mathrm{v}}^{ \pm}, \Gamma_{\mathrm{h}}^{ \pm}$, there is at least one such family with the property that $\gamma$ intersects every path in this family. Since extremal lengths of horizontal and vertical path families in rectangles are reciprocal quantities, we have

$$
\Lambda(h(\Gamma)) \geq \frac{1}{k}
$$

and the proof of the bound is complete.

\section{References}

[Cui] G. Z. Cui, Geometrically finite rational maps with given combinatorics, manuscript, 2000.

[GL] F. P. Gardiner and N. Lakic, Quasiconformal Teichmüller Theory, Math. Surveys Monogr. 76, Amer. Math. Soc., Providence, RI, 2000.

[GK] L. R. Goldberg and L. Keen, The mapping class group of a generic quadratic rational map and automorphisms of the 2-shift, Invent. Math. 101 (1990), 335372.

[Haï] P. Haïssinsky, Pincement de polynômes, Comment. Math. Helv. 77 (2002), 1-23.

[K] J. Kahn, Holomorphic removability of Julia sets, preprint, ArXiv math.DS/ 9812164, 1998.

[Leh] O. Lehto, Univalent Functions and Teichmüller Spaces, Grad. Texts in Math. 109, Springer, New York, 1987.

[LV] O. Lehto and K. I. Virtanen, Quasiconformal Mappings in the Plane, 2nd ed., Grundlehren Math. Wiss. 126, Springer, New York, 1973.

[Mak] P. M. Makienko, Modular groups of certain rational functions, Dokl. Akad. Nauk SSSR 310 (1990), 793-794 (in Russian); English transl.: Soviet Math. Dokl. 41 (1990), 126-127.

[McM] C. T. McMullen, Hausdorff dimension and conformal dynamics. II. Geometrically finite rational maps, Comment. Math. Helv. 75 (2000), 535-593.

[MS] C. T. McMullen and D. P. Sullivan, Quasiconformal homeomorphisms and dynamics. III. The Teichmüller space of a holomorphic dynamical system, Adv. Math. 135 (1998), 351-395.

[Mil] J. Milnor, Dynamics in One Complex Variable, Vieweg, Braunschweig, 1999.

[PT] K. M. Pilgrim and L. Tan, Spinning deformations of rational maps, Conform. Geom. Dyn. 8 (2004), 52-86. 
[R] J. Rivera-Letelier, Rigid annuli, Thurston's pull-back argument and ColletEckmann condition, preprint, 2000.

Department of Mathematics

Indiana University

Bloomington, IN 47401, U.S.A.

E-mail: pilgrim@indiana.edu

Received 18 May 2003;

in revised form 14 June 2004 